\title{
Características e Tendências na Mortalidade em Diferentes Fenótipos de Insuficiência Cardíaca na Atenção Primária
}

\author{
Characteristics and Temporal Trends in the Mortality of Different Heart Failure Phenotypes in Primary Care \\ Antonio José Lagoeiro Jorge, ${ }^{10}$ Leticia Mara dos Santos Barbetta, ${ }^{10}$ Eduardo Thadeu de Oliveira Correia, ${ }^{10}$ Wolney \\ de Andrade Martins, ${ }^{10}$ Adson Renato Leite, ${ }^{1}$ Maria Auxiliadora Nogueira Saad, ${ }^{1}$ Márcia Maria Sales dos Santos, ${ }^{1}$ Dayse \\ Mary Correia, ${ }^{1}$ Maria Luiza Garcia Rosa, ${ }^{10}$ Sérgio Chermont, ${ }^{1}$ Cárita Cunha dos Santos, ${ }^{1}$ Evandro Tinoco Mesquita ${ }^{1,2}$ (은 \\ Universidade Federal Fluminense, ${ }^{1}$ Niterói, RJ - Brasil
}

C.T.E.B, UHG, ${ }^{2}-$ Brasil

\section{Resumo}

Fundamento: A classificação da insuficiência cardíaca (IC) por fenótipos possui grande relevância na prática clínica.

Objetivo: O estudo visou analisar a prevalência, as características clínicas e os desfechos entre os fenótipos de IC no contexto da atenção primária.

Métodos: Trata-se de uma análise de um estudo de coorte que incluiu 560 indivíduos, com idade $\geq 45$ anos, que foram selecionados aleatoriamente em um programa de atenção primária. Todos os participantes foram submetidos a avaliações clínicas, dosagem do peptídeo natriurético tipo B (BNP), eletrocardiograma e ecocardiografia em um único dia. A IC com fração de ejeção do ventrículo esquerdo (FEVE) < $40 \%$ foi classificado como IC com fração de ejeção reduzida (ICFEr), FEVE de $40 \%$ a $49 \%$ como IC com fração de ejeção intermediária (ICFEi) e FEVE $\geq 50 \%$ como IC com fração de ejeção preservada (ICFEp). Após 5 anos, os pacientes foram reavaliados quanto à ocorrência do desfecho composto de óbito por qualquer causa ou internação por doença cardiovascular.

Resultados: Dos 560 pacientes incluídos, 51 pacientes tinham IC (9,1\%), 11 dos quais tinham ICFEr (21,6\%), 10 tinham ICFEi (19,6\%) e 30 tinham ICFEp (58,8\%). A ICFEi foi semelhante à ICFEp nos níveis de BNP (p < 0,001), índice de massa do ventrículo esquerdo $(p=0,037)$ e índice de volume do átrio esquerdo $(p<0,001)$. O fenótipo de ICFEi foi semelhante ao de ICFEr em relação à doença arterial coronariana $(p=0,009)$. Após 5 anos, os pacientes com ICFEi apresentaram melhor prognóstico quando comparados aos pacientes com ICFEp e ICFEr $(p<0,001)$.

Conclusão: A prevalência de ICFEI foi semelhante ao observado em estudos anteriores. A ICFEI apresentou características semelhantes a ICFEP neste estudo. Nossos dados mostram que a ICFEi teve melhor prognóstico em comparação com os outros dois fenótipos.

Palavras-chave: Insuficiência Cardíaca/tendências; Insuficiência Cardíaca/mortalidade; Prevalência; Atenção Primária a Saúde; Prognóstico; Epidemiologia; Volume Sistólico.

\footnotetext{
Abstract

Background: The classification of heart failure (HF) by phenotypes has a great relevance in clinical practice.

Objective: The study aimed to analyze the prevalence, clinical characteristics, and outcomes between HF phenotypes in the primary care setting

Methods: This is an analysis of a cohort study including 560 individuals, aged $\geq 45$ years, who were randomly selected in a primary care program. All participants underwent clinical evaluations, b-type natriuretic peptide (BNP) measurements, electrocardiogram, and echocardiography in a single day. HF with left ventricular ejection fraction (LVEF) < 40\% was classified as HF with reduced ejection fraction (HFrEF), LVEF $40 \%$ to $49 \%$ as HF with mid-range ejection fraction (HFmrEF) and LVEF $\geq 50 \%$ as HF with preserved ejection fraction (HFpEF). After 5 years, the patients were reassessed as to the occurrence of the composite outcome of death from any cause or hospitalization for cardiovascular disease.

Results: Of the 560 patients included, 51 patients had HF (9.1\%), 11 of whom had HFrEF (21.6\%), 10 had HFmrEF (19.6\%) and 30 had HFPEF (58.8\%). HFmrEF was similar to HFpEF in BNP levels $(p<0.001)$, left ventricular mass index $(p=0.037)$, and left atrial volume index $(p<0.001)$. The HFmrEF phenotype was similar to HFrEF regarding coronary artery disease $(p=0.009)$. After 5 years, patients with HFmrEF had a better prognosis when compared to patients with HFpEF and HFrEF $(p<0.001)$.
}

Correspondência: Antonio José Lagoeiro Jorge •

Universidade Federal Fluminense - Rua Marques de Paraná, 303. CEP 24033-900, Centro, Niterói, RJ - Brasil

E-mail: lagoeiro@globo.com

Artigo recebido em 28/12/2019, revisado em 25/06/2020, aceito em 12/08/2020

DOI: https://doi.org/10.36660/abc.20190912 
Conclusion: The prevalence of ICFEI was similar to that observed in previous studies. ICFEI presented characteristics similar to ICFEP in this study. Our data show that ICFEi had a better prognosis compared to the other two phenotypes.

Keywords: Heart Failure/trends; Heart Failure/mortality; Prevalence; Primary Health Care; Prognosis; Eidemiology; Stroke Volume.

Full texts in English - http://www.arquivosonline.com.br

\section{Introdução}

A classificação da insuficiência cardíaca (IC) por fenótipos possui grande relevância na prática clínica, uma vez que diferem em relação às características, ao prognóstico e ao tratamento do paciente. ${ }^{1}$ Classicamente, dois fenótipos de IC foram descritos nas diretrizes, a saber, IC com fração de ejeção reduzida (ICFEr) onde a fração de ejeção do ventrículo esquerdo (FEVE) é inferior a 50\% e IC com fração de ejeção preservada (ICFEp) com FEVE $\geq 50 \%{ }^{2}$ Em 2013, a American College of Cardiology Foundation/American Heart Association publicou novas diretrizes para IC, nas quais os pacientes com FEVE entre $41 \%$ e $50 \%$ foram classificados como casos limítrofes de ICFEp. ${ }^{3}$ Em 2016, as diretrizes de IC da Sociedade Europeia de Cardiologia reconheceram a IC com FEVE entre $40 \%$ e $49 \%$ como um fenótipo distinto, denominado IC com fração de ejeção intermediária (ICFEi). ${ }^{4}$ Finalmente, em 2018, a Sociedade Brasileira de Cardiologia incluiu a ICFEi nas Diretrizes de Insuficiência Cardíaca Crônica e Aguda de $2018 .^{5}$

Estudos recentes observaram que a prevalência de pacientes com ICFEi variou de $13 \%$ a $24 \%$ de todos os pacientes com IC. ${ }^{6-8}$ Dados atuais de estudos de IC indicam que a ICFEi apresenta características intermediárias. ${ }^{8}$ Além disso, uma metanálise que incluiu mais de 600.000 pacientes com IC concluiu que os pacientes com ICFEi apresentaram mortalidade por todas as causas mais baixa do que os pacientes com ICFEr e nenhuma diferença estatística dos pacientes com ICFEp. Em relação às internações por todas as causas, não houve diferença estatística entre os três fenótipos de IC. ${ }^{9}$

Não há estudos no Brasil que avaliem esse fenótipo na atenção primária. Portanto, o presente estudo visou analisar a prevalência e as características clínicas da ICFEi, bem como os desfechos entre os fenótipos de IC em pacientes no contexto da atenção primária.

\section{Métodos}

Este estudo de coorte incluiu, na linha de base, 633 indivíduos com idade $\geq 45$ anos, cadastrados no Programa de Atenção Primária do município de Niterói, município de médio porte com 487.562 habitantes no estado do Rio de Janeiro, Brasil. O Programa de Atenção Básica oferece cobertura a 137.463 residentes em 32 módulos de atendimento, divididos em 110 setores. Inicialmente, foram selecionados 21 setores por meio de uma sequência aleatória, gerada por um programa de computador, em que o peso de cada setor era proporcional ao número de indivíduos. ${ }^{10}$ Os dados foram coletados de julho de 2011 a dezembro de 2012. Após 5 anos, os pacientes neste estudo foram reavaliados quanto à ocorrência do desfecho composto de óbito por qualquer causa ou internação por doença cardiovascular. Durante o seguimento, ocorreram 73 (11,5\%) perdas e o número final de indivíduos avaliados foi 560.

\section{População}

O tamanho da amostra foi estimado a partir de uma prevalência mínima de IC de $6 \%$, com um erro absoluto de $2 \%$ (intervalo de confiança $=99 \%, 4 \%$ a $8 \%$ ). Tal suposição requeria um tamanho de amostra de 580 indivíduos. Em cada um dos 21 setores incluídos, foram aleatoriamente selecionados 30 indivíduos entre 45 e 100 anos de idade. Foram escolhidos 20 indivíduos adicionais por unidade, para permitir a substituição em caso de impossibilidade de participação, totalizando 1.050 indivíduos selecionados. Dessa forma, enviamos cartas aos funcionários das unidades de saúde para convidar 1.050 indivíduos a participarem deste estudo, sendo que 666 desses indivíduos compareceram à consulta e assinaram o termo de consentimento livre e esclarecido. Foram excluídos 33 indivíduos que não completaram todos os procedimentos da pesquisa. A população de base era de 633 indivíduos, no entanto, 73 (11,5\%) não foram localizados após 5 anos e foram subsequentemente excluídos. A população final foi de 560 indivíduos. (Figura 1)

A escolha das unidades básicas de saúde e o número de indivíduos em cada unidade foram planejados de forma a representar a distribuição demográfica. A seleção dos sujeitos foi realizada por meio de uma sequência aleatória gerada por um programa de computador. Os critérios de inclusão foram idade $\geq 45$ anos e disponibilidade em dar consentimento informado. Sempre que ocorria uma recusa, o próximo sujeito da lista aleatória era convidado a participar.

Todos os participantes do estudo foram submetidos a uma avaliação de um único dia que consistiu em: (a) anamnese e exames clínicos; (b) testes laboratoriais, incluindo dosagem do peptídeo natriurético do tipo B (BNP); (c) eletrocardiograma (ECG) de 12 derivações; e (d) ecocardiografia Doppler tecidual. Foi realizado o ECG em 12 derivações simultâneas. A ecocardiografia com Doppler tecidual foi realizada por dois médicos certificados, utilizando dois aparelhos portáteis, o Acuson Cypress 20 (Siemens, EUA) e o AU-3 Partner (Esaote, Itália). Os médicos foram cegados ao estado clínico e aos resultados dos exames. Os exames foram realizados de acordo com as recomendações de quantificação de câmaras da Sociedade Americana de Ecocardiografia e da Associação Europeia de Ecocardiografia. ${ }^{11}$ A função sistólica foi avaliada medindo a FEVE usando o método de Simpson.

\section{Definição dos fenótipos de insuficiência cardíaca}

Foi confirmado o diagnóstico de ICFEr em indivíduos com histórico de IC ou a presença de sinais ou sintomas de IC e FEVE $<40 \%$. Foi confirmado o diagnóstico de ICFEp em indivíduos com histórico de IC ou sinais ou sintomas de IC com FEVE $\geq 50 \%$ e índice de volume diastólico final (IVDF) $<$ $97 \mathrm{~mL} / \mathrm{m}^{2}$, na presença de disfunção diastólica do ventrículo esquerdo e BNP > $35 \mathrm{pg} / \mathrm{mL}$. A ICFEi foi confirmada em 


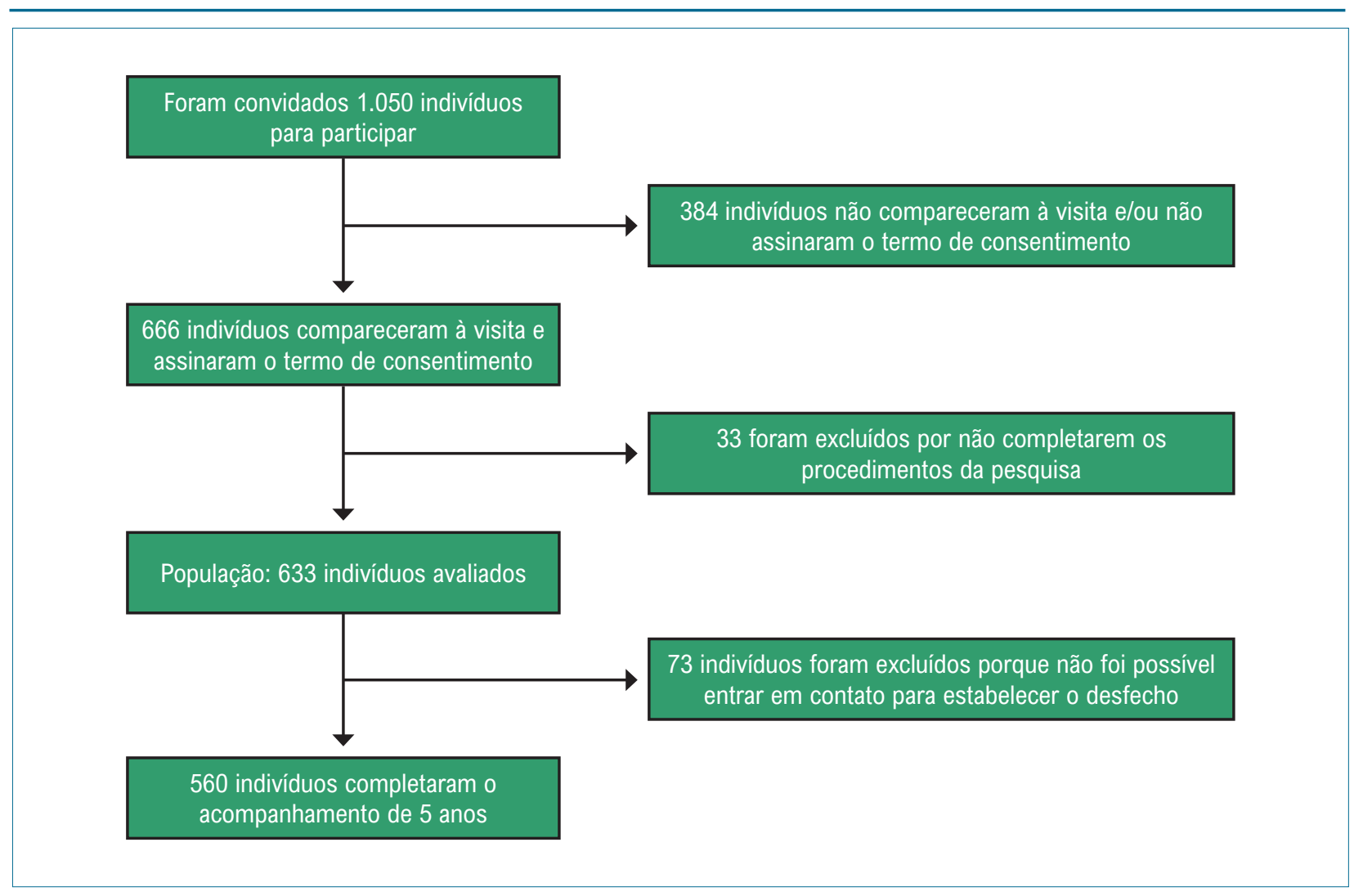

Figura 1 - Fluxograma de seleção da população

indivíduos com histórico de IC ou sinais ou sintomas de IC com FEVE entre $40 \%$ e $49 \%$ e BNP > 35 pg/mL. ${ }^{4,12}$

\section{Análise estatística}

As variáveis contínuas foram expressas como mediana e intervalo interquartil, uma vez que nenhuma delas foi positiva para normalidade quando testada pelo teste de Kolmogorov-Smirnov. As variáveis categóricas foram resumidas como frequências absolutas e relativas. Em relação às variáveis quantitativas, as diferenças entre os fenótipos de IC foram testadas com os testes não paramétricos (Mann-Whitney e Kruskal-Wallis), enquanto as variáveis categóricas foram avaliadas pelo teste de quiquadrado. Foi estimada uma curva de Kaplan-Meier para os desfechos compostos das quatro possibilidades (sem IC, ICFEr, ICFEi e ICFEp). A diferença entre as quatro curvas e entre o grupo ICFEi e o grupo sem IC foi testada com o teste de log rank. Foram considerados estatisticamente significativos valores de $p<0,05$. Todas as análises estatísticas foram realizadas com o software SPSS versão 23.0 (Chicago, Illinois, EUA).

\section{Considerações éticas}

O presente estudo foi realizado de acordo com os princípios da Declaração de Helsinque revisada em 2000. O protocolo do estudo foi aprovado pelo Comitê de Ética institucional sob o número 0077.0.258.000-10.

\section{Resultados}

\section{Prevalência e características de pacientes com ICFEi}

Dos 560 pacientes incluídos no estudo, 509 não tinham o diagnóstico de IC (90,9\%) e 51 foram diagnosticados com IC (9,1\%). Dos 51 pacientes com IC, 11 tinham $\operatorname{ICFEr}(21,6 \%), 10$ tinham ICFEi (19,6\%) e 30 tinham ICFEp (58,8\%). As características demográficas e clínicas dos pacientes com IC são apresentadas na Tabela 1. A ICFEi foi semelhante à ICFEp em relação ao índice de massa do ventrículo esquerdo (IMVE) e ao índice de volume do átrio esquerdo (IVAE). Observamos mais doença arterial coronariana em pacientes com fenótipo ICFEr, em comparação com ICFEi. O percentual de doença renal crônica foi intermediário no grupo ICFEi, sendo inferior ao grupo ICFEp e superior ao grupo ICFEr. O grupo ICFEi apresentou valores intermediários nas seguintes características: frequência cardíaca, glicemia e relação creatinina-albumina. Porém, não houve diferença estatística entre os grupos com IC com relação a essas características.

Ao analisar os parâmetros ecocardiográficos, a razão E/e' média, o IMVE, o IVAE e o IVDF apresentaram diferença estatística na análise geral, com $p<0,001$ em todas as análises. Os IMVE, IVAE e IVDF apresentaram valores intermediários no grupo ICFEi. O IMVE no grupo ICFEi foi menor do que no grupo ICFEr e semelhante ao 
Tabela 1 - Características demográficas e clínicas de pacientes com insuficiência cardíaca, de acordo com fenótipo ICFEp, ICFEi ou ICFEp

\begin{tabular}{|c|c|c|c|c|c|c|c|c|}
\hline & Sem IC (n=509) & ICFEr (n=11) & ICFEi $(n=10)$ & ICFEp (n=30) & Geral & $\begin{array}{l}\text { ICFEr vs. } \\
\text { ICFEi }\end{array}$ & $\begin{array}{l}\text { ICFEp vs. } \\
\text { ICFEi }\end{array}$ & $\begin{array}{l}\text { ICFEr vs. } \\
\text { ICFEp }\end{array}$ \\
\hline $\begin{array}{l}\text { Sexo masculino } \\
(\%)\end{array}$ & 37 & 64 & 40 & 27 & 0,190 & 0,279 & 0,426 & 0,029 \\
\hline $\begin{array}{l}\text { Idade, anos } \\
\text { (mediana) }\end{array}$ & $57(51-64)$ & $74(57-78)$ & $72(60-79)$ & $72,5(64,7-81,7)$ & $<0,001$ & 0,809 & 0,708 & 0,871 \\
\hline IMC (mediana) & $27,2(24,5-30,8)$ & $24,9(21,3-25,9)$ & $28,1(26,3-30,6)$ & $26,9(22,0-30,7)$ & 0,156 & 0,057 & 0,319 & 0,496 \\
\hline $\begin{array}{l}\mathrm{FC}, \text { bpm } \\
\text { (mediana) }\end{array}$ & $70,5(63,2-77,5)$ & $69(55,5-72,5)$ & $72(62,1-79,1)$ & $76,5(63,2-84,7)$ & 0,360 & 0,324 & 0,573 & 0,108 \\
\hline $\begin{array}{l}\text { PA sistólica, } \\
\text { mmHg (mediana) }\end{array}$ & $\begin{array}{c}133,3(121- \\
147,5)\end{array}$ & 146(116-161) & $130(117,9-157,8)$ & $\begin{array}{c}151,7(135,2- \\
179,7)\end{array}$ & 0,001 & 0,751 & 0,032 & 0,168 \\
\hline $\begin{array}{l}\text { PA diastólica, } \\
\text { mmHg (mediana) }\end{array}$ & $82(74,1-90)$ & $80(68,3-88,5)$ & $77,5(71,1-90,9)$ & $83,7(72,7-91,3)$ & 0,699 & 0,778 & 0,699 & 0,310 \\
\hline $\begin{array}{l}\text { BNP, pg/mL } \\
\text { (mediana) }\end{array}$ & $15(10-25)$ & $306(153-615)$ & $61,5(51-95)$ & $87,5(52,7-120,5)$ & $<0,0001$ & 0,002 & 0,281 & 0,001 \\
\hline $\begin{array}{l}\text { Glicose, mg/dL } \\
\text { (mediana) }\end{array}$ & 100(91-113) & 103(84-119) & $97(87-106,2)$ & $100(94,7-119)$ & 0,765 & 0,621 & 0,288 & 0,757 \\
\hline $\begin{array}{l}\text { Ácido úrico, mg/ } \\
\mathrm{dL} \text { (mediana) }\end{array}$ & $5,1(4,2-6,1)$ & $6,3(4,6-8,0)$ & $5,2(4,9-6,5)$ & $5,1(4,1-6,7)$ & 0,192 & 0,398 & 0,430 & 0,108 \\
\hline $\begin{array}{l}\text { Colesterol total, } \\
\text { mg/dL (mediana) }\end{array}$ & 213(186-244) & $185(177-253)$ & 199(180-240) & $208(196-231)$ & 0,629 & 0,623 & 0,453 & 0,502 \\
\hline $\begin{array}{l}\text { Triglicerídeos, mg/ } \\
\text { dL (mediana) }\end{array}$ & 118(86-169) & $115(86-190)$ & $106(66-152)$ & $101(90-136)$ & 0,481 & 0,571 & 0,851 & 0,482 \\
\hline $\begin{array}{l}\text { Hemoglobina, g/ } \\
\mathrm{dL} \text { (mediana) }\end{array}$ & $13,7(12,8-14,7)$ & $13,9(13,4-16,4)$ & $13,7(12,1-14,3)$ & $13,9(12,6-14,7)$ & 0,396 & 0,204 & 0,370 & 0,435 \\
\hline $\begin{array}{l}\text { Microalbuminúria, } \\
\mathrm{mg} / \mathrm{L} \text { (mediana) }\end{array}$ & $11,2(5,9-23,4)$ & $29,5(10,1-58,7)$ & $11,1(3,9-31,1)$ & $14,3(6,6-38,3)$ & 0,265 & 0,178 & 0,457 & 0,371 \\
\hline $\begin{array}{l}\text { TFGe, } \mathrm{mL} / \\
\mathrm{min} / 1,73 \mathrm{~m}^{2} \\
\text { (mediana) }\end{array}$ & $83,5(71,6-96,1)$ & $76,3(47-103,1)$ & $84,1(52,7-100,7)$ & $69,4(50,5-89,1)$ & 0,009 & 0,888 & 0,303 & 0,427 \\
\hline $\begin{array}{l}\mathrm{RCA}, \mathrm{mg} / \mathrm{g} \\
\text { (mediana) }\end{array}$ & $9,7(5,6-22,4)$ & $40,1(7,8-78,5)$ & $19,8(5,9-33,3)$ & $15,7(8,6-45,2)$ & 0,051 & 0,270 & 0,821 & 0,385 \\
\hline Diabetes (\%) & 24 & 27 & 0 & 27 & 0,341 & 0,074 & 0,068 & 0,969 \\
\hline Hipertensão (\%) & 70 & 91 & 90 & 90 & 0,028 & 0,943 & 1,000 & 0,931 \\
\hline DAC (\%) & 7,5 & 27 & 10 & 27 & 0,001 & 0,314 & 0,274 & 0,969 \\
\hline DRC (\%) & 8,9 & 27,3 & 40 & 33,3 & $<0,0001$ & 0,537 & 0,702 & 0,712 \\
\hline IECA/BRA (\%) & 38 & 64 & 70 & 47 & 0,184 & 0,757 & 0,411 & 0,565 \\
\hline $\begin{array}{l}\text { Betabloqueadores } \\
(\%)\end{array}$ & 14 & 36 & 30 & 30 & 0,012 & 0,757 & 1,000 & 0,698 \\
\hline Diuréticos (\%) & 34 & 36 & 50 & 53 & 0,148 & 0,528 & 0,855 & 0,335 \\
\hline $\begin{array}{l}\text { Desfecho } \\
\text { composto, n (\%) }\end{array}$ & $39(7,7)$ & $7(63,6)$ & $3(30)$ & $15(50)$ & $<0,0001$ & 0,123 & 0,271 & 0,438 \\
\hline
\end{tabular}

BNP: peptídeo natriurético tipo B; bpm: batimentos por minuto; BRA: bloqueador do receptor de angiotensina; DAC: doença arterial coronariana; DRC: doença renal crônica; FC: frequência cardíaca; IC: insuficiência cardíaca; ICFEi: insuficiência cardíaca com fração de ejeção intermediária; ICFEp: insuficiência cardíaca com fração de ejeção preservada; ICFEr: insuficiência cardíaca com fração de ejeção reduzida; IECA: inibidor da enzima de conversão da angiotensina; IMC: índice de massa corporal; PA: pressão arterial; RCA: relação creatinina-albumina; TFGe: taxa de filtração glomerular estimada. As variáveis categóricas são apresentadas como porcentagem (\%) e as variáveis contínuas como mediana e intervalo interquartil (25\% e 75\%); o valor p geral para variáveis contínuas foi calculado com o teste de Kruskal-Wallis; as diferenças entre ICFEp, ICFEi e ICFEr foram calculadas usando o teste de Mann-Whitney; os valores de p para variáveis categóricas foram calculados usando o qui-quadrado de Pearson.

grupo ICFEp. O IVAE no grupo ICFEi foi significativamente menor do que no grupo ICFEr e semelhante ao grupo ICFEp. O IVDF foi mais alto no grupo ICFEi quando comparado ao grupo ICFEp e menor quando comparado ao grupo ICFEr. Além disso, quando a razão E/e' média em ICFEi e ICFEr foram analisados separadamente, a razão $\mathrm{E} / \mathrm{e}^{\prime}$ do grupo ICFEi foi menor do que a do grupo ICFEr. (Tabela 2) 
Tabela 2 - Características clínicas de pacientes com insuficiência cardíaca, de acordo com fenótipo ICFEp, ICFEi ou ICFEp

\begin{tabular}{lcccccccc}
\hline & Sem IC (n=509) & ICFEr $(\mathbf{n = 1 1 )}$ & ICFEi $(\mathbf{n = 1 0 )}$ & ICFEp $(\mathbf{n = 3 0 )}$ & Geral & $\begin{array}{c}\text { ICFEr vs. } \\
\text { ICFEi }\end{array}$ & $\begin{array}{c}\text { ICFEp } \\
\text { vs. ICFEi }\end{array}$ & $\begin{array}{c}\text { ICFEr vs. } \\
\text { ICFEp }\end{array}$ \\
\hline Fração de ejeção, $\%$ & $61(58-65)$ & $29(23-33)$ & $43,5(41-48)$ & $59,5(56,7-64,2)$ & $<0,0001$ & $<0,0001$ & $<0,0001$ & $<0,0001$ \\
\hline Razão E/e' média, $( \pm S D)$ & $6,5(5,4-7,8)$ & $9,6(7,5-17)$ & $8,3(6-9,1)$ & $7,9(6,1-12,1)$ & $<0,0001$ & 0,149 & 0,791 & 0,162 \\
\hline IVAE, $\mathrm{ml} / \mathrm{m}^{2},( \pm S D)$ & $20,9(17,3-24,5)$ & $38,6(26,8-65,9)$ & $30,5(18,9-42,2)$ & $29,4(24,3-41,8)$ & $<0,0001$ & 0,231 & 0,607 & 0,188 \\
\hline IMVE, $\mathrm{g} / \mathrm{m}^{2},( \pm S D)$ & $89,3(76,5-102,8)$ & $160,2(113,1-187,3)$ & $119,0(102,9-154,0)$ & $104,2(76,9-127,1)$ & $<0,0001$ & 0,091 & 0,123 & 0,002 \\
\hline IVDF, $\mathrm{ml} / \mathrm{m}^{2},( \pm S D)$ & $62,8(54,5-71,2)$ & $106,0(82,5-150,3)$ & $93,8(75,6-114,3)$ & $68,7(54,2-76,2)$ & $<0,0001$ & 0,360 & $<0,0001$ & 0,001 \\
\hline
\end{tabular}

E: velocidade de fluxo mitral precoce; E': velocidade diastólico precoce do anel mitral; IC: insuficiência cardíaca; ICFEi: insuficiência cardíaca com fração de ejeção intermediária; ICFEp: insuficiência cardiaca com fração de ejeção preservada; ICFEr: insuficiência cardíaca com fração de ejeção reduzida; IVAE: índice de volume do átrio esquerdo; IVDF: índice de volume diastólico final; IMVE: índice de massa do ventrículo esquerdo. Os dados são apresentados como mediana e intervalo interquartil (25\% e $75 \%)$; ( ${ }^{*}$ ) o valor p geral foi calculado pelo teste de Kruskal-Wallis; as diferenças entre ICFEp, ICFEi e ICFEr foram calculadas usando o teste de Mann-Whitney.

\section{Prognóstico de fenótipos de IC}

Após 5 anos, ocorreram 64 desfechos compostos, especificamente, 50 óbitos e 14 internações hospitalares por doença cardiovascular. Na curva de Kaplan-Meier (Figura 2), os pacientes com ICFEi apresentaram pior desfecho composto de óbito por todas as causas e interação cardiovascular do que os pacientes sem IC. No entanto, os pacientes com ICFEi tiveram melhor prognóstico na análise de Kaplan-Meier, quando comparados aos pacientes com ICFEp e ICFEr, enquanto os pacientes com ICFEp tiveram melhor prognóstico do que aqueles com fenótipo ICFEr. A Tabela 3 mostra as médias e seus intervalos de confiança de sobrevivência para os diferentes fenótipos de IC.

\section{Discussão}

Desde a adoção de ICFEi como um novo fenótipo de IC, o desafio maior tem sido definir as características de linha de base, a fisiopatologia e o tratamento para este novo grupo de pacientes. O presente artigo é o primeiro estudo do fenótipo de ICFEi em uma população brasileira, envolvendo pacientes da atenção primária. Realizamos uma análise do estudo Digitalis ${ }^{10} \mathrm{Com}$ a finalidade de avaliar a prevalência e as características clínicas e ecocardiográficas de pacientes com ICFEi no Brasil.

Em nossa população de pacientes com IC, a prevalência de ICFEi foi de $22 \%$, semelhante a outros estudos. ${ }^{6-8}$

Os estudos de Rickenbacher et al. ${ }^{12}$ e Tsuji et al.,' mostraram que os níveis de BNP eram intermediários na ICFEi. Porém, em nosso estudo, o BNP no grupo ICFEi não apresentou valores intermediários; foi semelhante ao grupo ICFEp e apresentou valores inferiores aos do grupo ICFEr. Porém, em relação à prevalência de etiologia isquêmica no grupo ICFEi, nosso estudo mostrou que ICFEi foi semelhante a ICFEr, semelhante a estudos anteriores. Resultados do estudo de Kapoor et al. ${ }^{6}$ e o registro sueco de $\mathrm{IC}^{11}$ sugerem que a etiologia isquêmica é distintamente mais comum na ICFEr e na ICFEi. O estudo TOPCAT ${ }^{13}$ avaliou o uso da espironolactona em pacientes com diferentes faixas de FEVE e mostrou que houve redução de internações em pacientes com IC, principalmente aqueles com FEVE entre $45 \%$ e $50 \%$. No estudo $\mathrm{CHARM}^{14}$ concluiu que o uso de candesartana melhorou os desfechos, tanto para ICFEi quanto para ICFEr. Assim, por extrapolação, ICFEi poderia responder ao tratamento recomendado para ICFEr de etiologia isquêmica, conforme sugerido pelas diretrizes do IC. ${ }^{3,5}$

Ao analisar os parâmetros da Doppler ecocardiografia, o IMVE, o IVAE e a razão E/e', no grupo ICFEi, foram semelhantes ao grupo ICFEp, enquanto o IVDF no grupo ICFE apresentou valores intermediários, com diferenças estatísticas quando comparado aos grupos ICFEp e ICFEr. O estudo de Rastogi et al., ${ }^{15}$ sugere que os pacientes com ICFEi são um grupo heterogêneo, com pelo menos 3 subgrupos com base na FEVE, a saber, pacientes com FEVE prévia $<40 \%$ (fração de ejeção recuperada), pacientes com FEVE prévia > 50\% (fração de ejeção deteriorada) e pacientes com FEVE prévia entre $40 \%$ e $50 \%$ (fração de ejeção inalterada). Esses achados reforçam a ideia de que a fisiopatologia da ICFEi pode ter uma contribuição da disfunção sistólica e uma contribuição da disfunção diastólica, conforme sugerido pelas diretrizes da Sociedade Europeia de Cardiologia de $2016 .{ }^{4}$

Em relação ao prognóstico, nosso estudo concluiu que os pacientes com ICFEi apresentaram melhor desfecho composto de mortalidade por todas as causas e internação cardiovascular melhor do que aqueles com ICFEr e ICFEp $(p<0,001)$. Nossos resultados estão de acordo com uma meta-análise de Altaie et al. ${ }^{9}$ que mostrou que o fenótipo ICFEi teve uma taxa de mortalidade por todas as causas significativamente menor do que ICFEr (RR, 0,9; intervalo de confiança de 95\%, 0,85 a 0,94; $p<0,001)$. No entanto, diferentemente do presente estudo, não verificaram nenhuma diferença significativa na mortalidade por todas as causas entre ICFEp e ICFEi (RR, 0,98; intervalo de confiança de $95 \%, 0,86$ a 1,12; $p=0,82) .{ }^{9}$ Na meta-análise por Altaie et al., analisando internação devido à IC, não encontraram diferenças significativas entre ICFEr e ICFEi (RR, 0,92; intervalo de confiança de 95\%, 0,84 a 1,01; $p=0,08$ ) ou entre ICFEp e ICFEi (RR, 1,05; intervalo de confiança de $95 \%, 0,83$ a 1,$33 ; p=0,69)$.

São necessários futuros estudos que investiguem o prognóstico e caracterizem a ICFEi com uma amostra maior. Além disso, o presente estudo abre caminho para futuros ensaios clínicos randomizados que investiguem tratamentos específicos para pacientes com ICFEi. 




Figura 2 - Curvas de Kaplan-Meier mostram que pacientes com ICFEi tiveram um pior desfecho composto de óbito por todas as causas e internação cardiovascular do que pacientes sem IC $(p<0,007)$, mas pacientes com ICFEi tiveram melhor prognóstico em comparação com pacientes com ICFEp e ICFEr $(p<0,001)$. O ICFEr teve o pior prognóstico dos três fenótipos de IC.

IC: insuficiência cardíaca; ICFEi: IC com fração de ejeção intermediária; ICFEp: IC com fração de ejeção preservada; ICFEr: IC com fração de ejeção reduzida.

Tabela 3 - Média e intervalo de confiança das probabilidades de sobrevivência em fenótipos de insuficiência cardíaca

\begin{tabular}{lccc}
\hline & & \multicolumn{2}{c}{ Intervalo de confiança 95\% } \\
\hline Variáveis & Estimativa média & Limite inferior & Limite superior \\
\hline Sem IC & 85,74 & 84,357 & 87,134 \\
\hline ICFEr & 41,81 & 25,646 & 57,990 \\
\hline ICFEi & 72,00 & 60,544 & 83,456 \\
\hline ICFEp & 54,56 & 45,561 & 63,572 \\
\hline
\end{tabular}

IC: insuficiência cardiaca; ICFEi: IC com fração de ejeção intermediária; ICFEp: IC com fração de ejeção preservada; ICFEr: IC com fração de ejeção reduzida.

\section{Limitações}

Os resultados devem ser interpretados com várias limitações. Primeiramente, foi avaliado um pequeno número de pacientes com IC, o que pode não representar toda a população. Em segundo lugar, a avaliação clínica e as variáveis laboratoriais e ecocardiográficas, incluindo a FEVE, foram baseadas em uma única medida. Além disso, embora as características sociodemográficas da população do estudo sejam bastante semelhantes às de outras áreas urbanas em todo o mundo, as extrapolações destes resultados devem ser feitas com cautela. Por fim, como a população do estudo era composta por voluntários, é possível que algum viés de seleção tenha sido introduzido, por exemplo, maior percentual de mulheres.

\section{Conclusão}

A prevalência de ICFEI foi semelhante ao observado em estudos anteriores. O presente estudo demonstrou que a ICFEi apresenta características clínicas e ecocardiográficas mais semelhantes à ICFEp, do que à ICFEr. Além disso, nossos dados mostram que a ICFEi teve melhor prognóstico em comparação com os outros dois fenótipos. 


\section{Contribuição dos autores}

Concepção e desenho da pesquisa: Jorge AJL, Barbetta LMS, Correia ETO, Rosa MLG, Mesquita ET; Obtenção de dados: Jorge AJL, Leite AR, Saad MAN, Correia DM, Chermont S; Análise e interpretação dos dados: Jorge AJL, Martins WA, Rosa MLG; Análise estatística: Rosa MLG, Santos CC; Redação do manuscrito: Jorge AJL, Barbetta LMS, Correia ETO; Revisão crítica do manuscrito quanto ao conteúdo intelectual importante: Jorge AJL, Martins WA, Mesquita ET, Santos MMS.

\section{Referências}

1. Wang TJ, Evans JC, Benjamin EJ, Levy D, LeRoy EC, Vasan RS. Natural history of asymptomatic left ventricular systolic dysfunction in the community. Circulation. 2003;108(8):977-82.

2. McMurray JJV, Adamopoulos S, Anker SD, Auricchio A, Böhm M, Dickstein $\mathrm{K}$, et al. ESC guidelines for the diagnosis and treatment of acute and chronic heart failure 2012: The Task Force for the Diagnosis and Treatment of Acute and Chronic Heart Failure 2012 of the European Society of Cardiology. Developed in collaboration with the Heart Failure Association (HFA) of the ESC. Eur J Heart Fail. 2012; 14(8):803-69.

3. Yancy CW, Jessup M, Bozkurt B, Butler J, Casey DE Jr, Drazner MH, et al. 2013 ACCF/AHA guideline for the management of heart failure: a report of the American College of Cardiology Foundation/American HeartAssociation Task Force on Practice Guidelines. J Am Coll Cardiol 2013;62(16):e147_ 239.

4. Ponikowski P, Voors AA, Anker SD, Bueno H, Cleland JGF, Coats AJS et al. 2016 ESC guidelines for the diagnosis and treatment of acute and chronic heart failure: the Task Force for the diagnosis and treatment of acute and chronic heart failure of the European Society of Cardiology (ESC) developed with the special contribution of the Heart Failure Association (HFA) of the ESC. Eur Heart J. 2016;37(27):2129-200

5. Comitê Coordenador da Diretriz de Insuficiência Cardíaca. Diretriz Brasileira de Insuficiência Cardíaca Crônica e Aguda.Sociedade Brasileira de Cardiologia. Arq Bras Cardiol. 2018; 111(3):436-539.

6. Kapoor JR, Kapoor R, Ju C, Heidenreich PA, Eapen ZJ, Hernandez AF et al. Precipitating clinical factors, heart failure characterization, and outcomes in patients hospitalized with heart failure with reduced, borderline, and preserved ejection fraction. J Am Coll Cardiol HF 2016;4(6):464-72.

7. Tsuji K, Sakata Y, Nochioka K, Miura M, Yamauchi T, Onose T et al. Characterization of heart failure patients with midrange left ventricular ejection fraction-a report from the CHART-2 study. Eur J Heart Fail. 2017;19(10):1258-69.

8. Lauritsen J, Gustafsson F, Abdulla J. Characteristics and long-term prognosis of patients with heart failure and mid-range ejection fraction compared
Potencial conflito de interesse

Não há conflito com o presente artigo

\section{Fontes de financiamento}

O presente estudo não teve fontes de financiamento externas.

\section{Vinculação acadêmica}

Não há vinculação deste estudo a programas de pós-graduação.

with reduced and preserved ejection fraction: A systematic review and meta-analysis. ESC Heart Fail 2018; 5(4): 685-94.

9. Altaie S, Khalife W. The prognosis of mid-range ejection fraction heart failure: a systematic review and meta-analysis. ESC Heart Fail. 2018;5(6):1008-16

10. Jorge AJL, Rosa MLG, Fernandes LCM, Freire MDC, Rodrigues RC, Correia DM da S, et al. Estudo da prevalência de insuficiência cardíaca em indivíduos cadastrados no Programa Médico de Família - Niterói. Estudo Digitalis: desenho e método. Rev Bras Cardiol. 2011;24;320-5.

11. Nagueh SF, Smiseth OA, Appleton CP, Byrd BF 3rd, Dokainish H, Edvardsen T,et al. Recommendations for the Evaluation of Left Ventricular Diastolic Function by Echocardiography: An Update from the American Society of Echocardiography and the European Association of Cardiovascular Imaging. J Am Soc Echocardiogr. 2016;29(4):277-314

12. Rickenbacher P, Kaufmann BA, Maeder MT Bernheim A, Goetschalckx K, Pfister $\mathrm{O}$, et al. Heart failure with mid-range ejection fraction: a distinct clinical entity? Insights from the Trial of Intensified versus standard Medical therapy in Elderly patients with Congestive Heart Failure (TIME-CHF). Eur J Heart Fail. 2017; 19:1586-1596

13. Koh AS, Tay WT, Teng TH, Vedin O, Benson L, Dahlstrom U, et al. A comprehensive population-based characterization of heart failure with mid-range ejection fraction. Eur J Heart Fail. 2017;19(12):1624-34.

14. Solomon SD, Claggett B, Lewis EF, Desai A, Anand I, Sweitzer NK, et al. Influence of ejection fraction on outcomes and efficacy of spironolactone in patients with heart failure with preserved ejection fraction. Eur Heart J 2016;37(5):455-62.

15. Lund LH, Claggett B, Liu J, Lam CS, Jhund PS, Rosano GM, et al. Heart failure with mid-range ejection fraction in CHARM: characteristics, outcomes and effect of candesartan across the entire ejection fraction spectrum. Eur J Heart Fail. 2018;20(8):1230-9.

16. Rastogi A, Novak E, Platts AE, Mann DL. Epidemiology, pathophysiology and clinical outcomes for heart failure patients with a mid-range ejection fraction. Eur J Heart Fail. 2017;19(12):1597-605. 